\title{
La hegemonía en cuestión: por una teoría del valor alternativa"
}

\author{
Nohora Angélica Sierra**, Luis Ricardo Martínez ${ }^{* * *}$, \\ Oscar Esteban Morillo ${ }^{* * * *}$
}

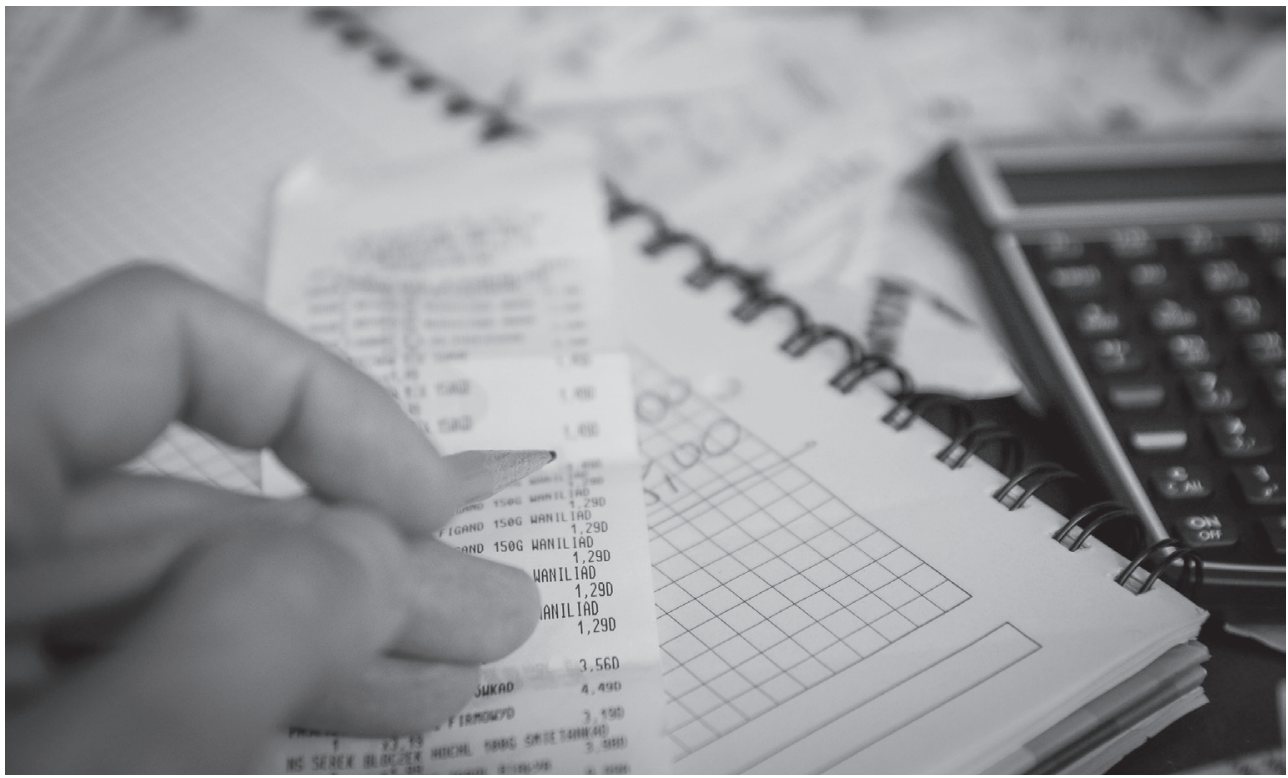

\section{Resumen}

En este artículo cuestionamos la ortodoxia de la teoría del valor frente a posibles alternativas. Hay una teoría económica dominante a través de la cual se nos presenta un mundo sujeto a leyes económicas incuestionables: la teoría neoclásica. Se hace necesaria una teoría del valor alternativa que permita dar cuenta de los

Citar este artículo como: Sierra, N. A., Martínez, L. R. y Morillo, O. E. (2020). La hegemonía en cuestión: por una teoría del valor alternativa. Revista Papeles, 11(22), 119-128.

Fecha de recibido: octubre 10 de 2019 • Fecha de aceptación: diciembre 5 de 2019

* El presente artículo toma como fuente principal las discusiones adelantadas por los autores como integrantes del Grupo de estudios de moneda, valor y dinero adscrito al Grupo de socioeconomía instituciones y desarrollo de la Universidad Nacional de Colombia (GSEID).

*: Investigadora, Antropóloga de la Universidad Nacional de Colombia, estudiante de maestría en antropología social en la Universidad Nacional de San Martín (Argentina). Correo electrónico: nasierraga@unal.edu.co

**: Investigador, Economista de la universidad Nacional de Colombia, estudiante de maestría en ciencias económicas en la Universidad Nacional de Colombia. Correo electrónico: omorillo@unal.edu.co

***: Investigador y estudiante del pregrado en Economía de la Universidad Nacional de Colombia, sede Bogotá. Correo electrónico: lurmartinezra@unal.edu.co 
mecanismos que posibilitan la coordinación de agentes descentralizados. Presentamos dos posturas frente a las teorías del valor alternativas que nos abren caminos para responder a la cuestión de la coordinación en una sociedad capitalista.

Palabras clave: teoría del valor, teoría neoclásica, teoría económica dominante.

Consideremos, para comenzar, la noción de fatalismo económico propuesta por Pierre Bourdieu (2000) en su discurso en Ludwigshafen, el 22 de noviembre de 1997. Según Bourdieu, se estaba dando, desde ese momento, un movimiento neoconservador cuyo propósito era imponer una doctrina económica que reduce toda práctica a las lógicas económicas; el contraataque neoliberal. En esencia, afirma Bourdieu, las leyes derivadas de las teorías neoliberales se muestran ahora como generales e ineludibles en todos los ámbitos de la existencia humana y, en la medida en que se acepta dicha doctrina como cierta, se moldean las realidades económicas a imagen y semejanza del credo neoliberal:

El neoliberalismo es una poderosa teoría económica cuya fuerza estrictamente simbólica, combinada con el efecto de la teoría, redobla la fuerza de las realidades económicas que supuestamente expresa (Bourdieu, 2000, p. 158).

Señala Bourdieu, también, a la teoría neoclásica de etnocéntrica, en tanto que esta busca, a fuerza de sus propios resultados, imponer premisas de acción dentro de propósitos universalizantes sobre las prácticas cotidianas, despojando a dichas prácticas de su singularidad social y cultural, y entendiéndolas de manera ahistórica.

Así, el fatalismo económico propone una narrativa que, sustentada en un conjunto de teorías económicas particulares, nos muestra un mundo que no puede ser de otra manera y que, a la vez, prescribe los cursos de acción a seguir que refuerzan el poder de dicha narrativa. En consecuencia, las leyes económicas son universales y no tiene sentido actuar en contra de ellas. En este sentido, si quisiéramos entender por qué las políticas de desarrollo no han sido capaces de combatir la desigualdad o el porqué de las recurrentes crisis económicas que redundan en el incremento de la pobreza en ciertos sectores de la sociedad, tendríamos que aceptar las premisas de la economía estándar, así como sus recetas. Después de todo, no hay más por hacer, el mundo es así y no hay otra explicación o alternativa.

Bourdieu utiliza los términos neoclásico y neoliberal para referirse a la misma cosa. En ellos recoge elementos que no se restringen al ámbito teórico, y que, sin embargo, incluyen la maximización de la utilidad, la autorregulación del mercado, el desarraigo de la teoría frente al contexto histórico y la inevitabilidad de las leyes que regulan la economía. De acuerdo con Colander (citado por Lozano, 2018), una teoría neoclásica tendrá, por lo menos, seis características, a saber: estudio de la asignación de recursos, supuestos de racionalidad perfecta $o$ casi perfecta, utilitarismo, análisis marginales, uso del individualismo metodológico y centro en el concepto de equilibrio. En adelante, usaremos el término neoclásico en este sentido, dado que, al momento de escribir este artículo, son las teorías neoclásicas, así connotadas, las que ocupaban el rol descrito por Bourdieu (2000) en 1997, y que, en nuestros días, son el paradigma dominante.

Las instituciones que gobiernan la acción pública y política emergen de un Estado influenciado y transformado por las ideas del paradigma dominante. De esta forma, surgen instituciones, fines y medios que representan el espíritu del proyecto político y social de esta hegemonía. Así, las instituciones, como medios para la "organización" de la realidad se definen al interior de la teoría neoclásica y se establecen como agentes de cambio y 
transformación de la conducta individual y colectiva.

En esta línea, la política pública se formula y se aplica teniendo en cuenta las necesidades concebidas por la teoría neoclásica. Siendo así que, por lo general, las formulaciones se centran en situaciones y formas que facilitan el desarrollo del mercado y, por extensión, de la mercantilización de la vida humana y su relación con el entorno. El mundo, para los economistas, se presenta entonces como un mundo en términos neoclásicos, pues se lee a través de una estructura institucional neoclásica. Este rol hegemónico que posibilita esta configuración del orden civil hace que la acción política se determine siguiendo los lineamientos del paradigma dominante $y$, de esta manera, dicho orden se reproduzca incansablemente.

Simplificando, los economistas neoclásicos, en ejercicio de su rol dominante, nos dicen que el mundo en el que vivimos es de una manera determinada, y que la forma que adopta el mundo es inescapable. Para saber esto, las teorías dominantes (la neoclásica en este caso) deberían ser capaces de explicar el mundo en que vivimos; esto es, deberían ser capaces de explicar el funcionamiento de una economía capitalista si pretende decirnos "cómo es", en efecto, dicha economía. Si, por el contrario, la economía neoclásica no pudiera cumplir con este requisito, aun así, ¿deberíamos aceptar sin más el fatalismo económico?

Para autores como Jean Cartelier (2018) y André Orléan (2014), la teoría neoclásica es incapaz de explicar una economía capitalista. Esta aseveración parte, en ambos casos, de la imposibilidad de la teoría neoclásica de explicar el funcionamiento de una economía de mercado descentralizada. Esta imposibilidad se funda, según estos dos autores, en la teoría del valor usada por la economía neoclásica.

Ahora bien, una teoría del valor se propone explicar cómo se forman los precios, cómo funciona la moneda y el dinero, cómo se explica la valoración de los bienes y cómo estos

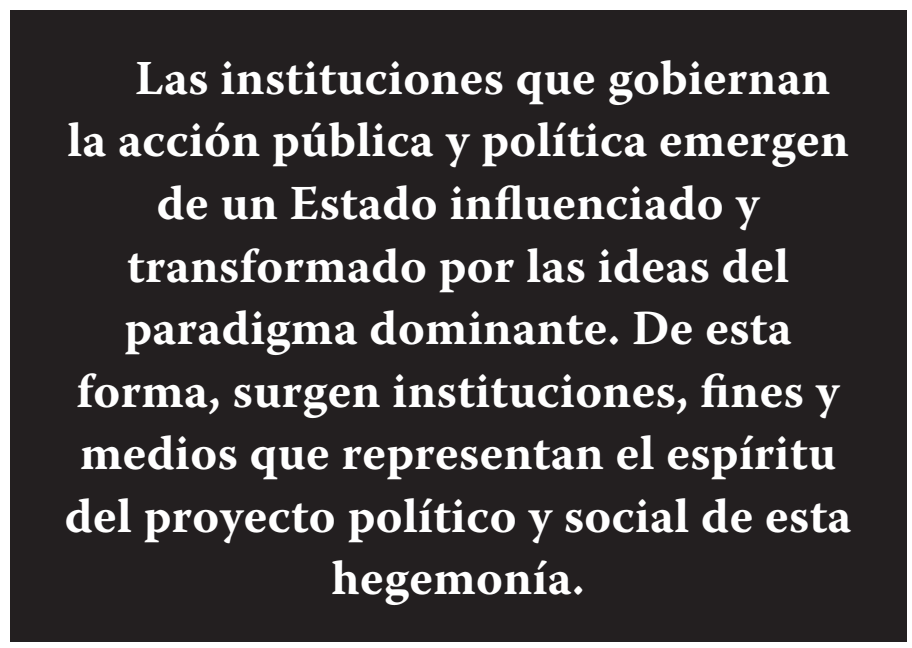

fenómenos se relacionan con la distribución de la riqueza. En este marco, la teoría neoclásica ha desarrollado, como máxima expresión de su teoría del valor, el modelo de equilibrio general walrasiano (EGW) que, según enuncia Debreu (1959) en su prefacio, se preocupa por:

1. Explicar el precio de las mercancías como resultado de la interacción de los agentes de una economía de propiedad privada a través del mercado.

2. Explicar el rol de los precios en un estado óptimo de la economía.

El análisis se organiza alrededor del concepto de un sistema de precios o, más generalmente, de una función de valor definida en el espacio de mercancías (p. 9).

Esta teoría ha sido presa de incontables críticas, la mayoría señalando la cuestión del realismo de la misma. Sin embargo, esta crítica carece de fuerza o pertinencia, sencillamente por el hecho de que una teoría no debe describir al pie de la letra la realidad, sino que se establece como una metáfora o parábola de la realidad (Screpanti y Zamagni, 1995). Teniendo en cuenta esto, debemos indagar por las preguntas que buscaba responder o aclarar dicha teoría en un principio. En este caso, la teoría neoclásica buscaba dar una solución lógica y coherente al problema de la coordinación mercantil planteado por Adam Smith. 
Adam Smith (1958) usó la metáfora de la "mano invisible" para describir la capacidad que tenía una sociedad mercantil, compuesta por individuos egoístas, de alcanzar un estado de armonía que fuera el más beneficioso para la colectividad (Tobin, 1992). Esta pregunta por la coordinación descentralizada de individuos se configuró como la pregunta central para la economía, y, por extensión, la pregunta por las condiciones que harían posible ese desarrollo armonioso.

Es bajo este marco que debemos evaluar la efectividad de la teoría neoclásica del EG para describir este proceso de coordinación, siendo que su intención como teoría no es describir la realidad, sino demostrar el proceso de ajuste de la economía hacia un equilibrio a través del mecanismo de precios propio de una sociedad mercantil. Recordando que el equilibrio es ese resultado estable y natural del proceso de agregación de decisiones individuales, es decir, el beneficio social de las acciones individuales egoístas (Smith, 1958).

La teoría neoclásica demostró la existencia del equilibrio; sin embargo, se encontró en un callejón sin salida (Ingrao e Israel, 1990) al momento de lograr demostrar y satisfacer las condiciones de estabilidad y de unicidad (resultados del teorema Sonnenschein-Mantel-Debreu). Esto implicó que la economía descrita por la teoría no fuera

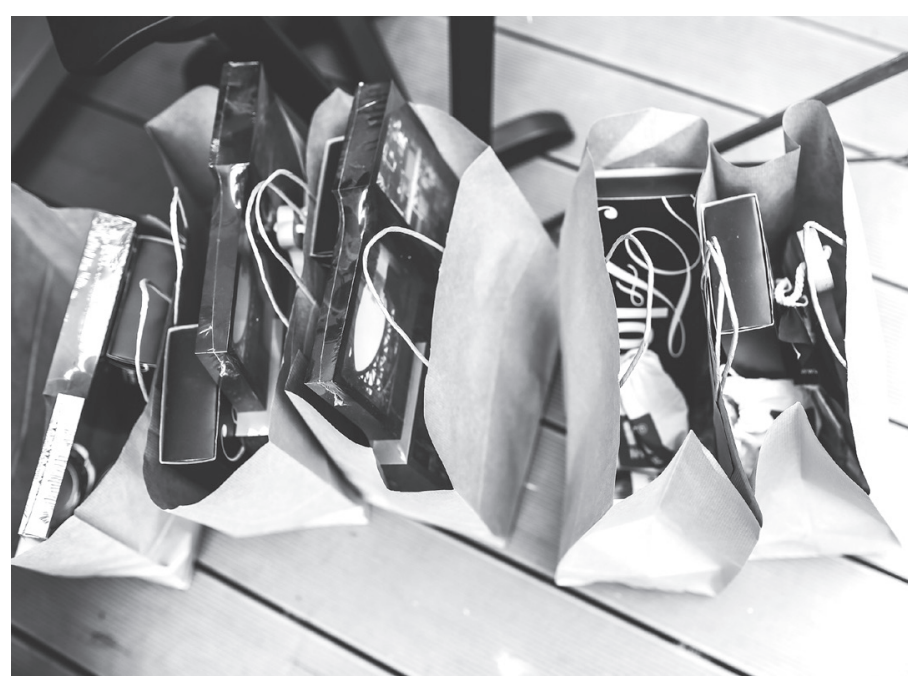

capaz de encontrar un trayecto estable hacia el equilibrio único y "natural" de Smith y que, a su vez, nada garantizara que el mecanismo de precios, propio de la economía de mercado, fuera el mecanismo apropiado para el proceso de ajuste hacia la convergencia.

Así, para solucionar la formación del vector de precios de equilibrio se sugirió una caja de compensación (Debreu, 1959) que registraba pagos entre individuos, y también un subastador benevolente que fijaría el vector de precios de equilibrio a partir del ejercicio de tanteo de precios. Esto significaba una excesiva centralización de la información (Rebeyrol, 1999) y una determinación, previa a toda interacción, de los precios (De Vroey, 1984). Pareciera entonces que el modelo de equilibrio general walrasiano propuesto por Arrow y Debreu fuera un modelo de absoluta centralización, similar a la planificación central del modelo socialista. De esta forma, se controvierte la idea del acceso al equilibrio a partir de una sociedad de mercado con individuos independientes, egoístas y libres.

La teoría neoclásica nos presenta un intento valioso de acercarse a responder la pregunta de Smith y sus condiciones; no obstante, sus resultados terminarían por demostrar casi lo contrario, al mismo tiempo que fueron incapaces de satisfacer sus ambiciones teóricas. Dicho todo esto, se nos hace evidente que el problema de la teoría neoclásica "no es la excesiva abstracción, sino el ser una mala abstracción" (Cataño, 2004, p. 199).

A pesar de este resultado, los neoclásicos no se dieron por vencidos y continuaron con la formulación, de mano de Arrow y Debreu, de lo que Benetti (1997) denomina el "método normativo", que consistía en establecer el EG como la norma, no solo para la formulación de recomendaciones de política económica sino para la explicación de las realidades económicas; esto es, la construcción de saber científico (Cataño, 2004).

$\mathrm{Su}$ origen se remonta a Arrow (1963), al referirse a las fallas de mercado como 
resultado de una desviación del equilibrio competitivo, implicando que todo análisis de las realidades económicas parte de la base del EG como un problema en la estructura de mercados, intuyéndose de esto que la solución de dicha fricción en los mercados permitiría el acercamiento de la realidad a ser como el modelo competitivo, lo cual se traduce en un estado social óptimo.

Por extensión, el método normativo que delimita la forma de entender la realidad y, por ende, de construir conocimiento, también será de radical importancia al momento de formular acciones de política pública que transformen la realidad, pues, partiendo del relajamiento de supuestos, se evalúan como situaciones subóptimas todas aquellas que no sean el EG competitivo, lo que se traducirá en una sugerencia de política que promueva la extensión del mercado y su liberalización, de manera que se permitirá un acercamiento a las condiciones del EG. Al respecto, resalta Benetti (1997):

el método normativo ofrece una respuesta: colocados en condiciones distintas a las del equilibrio general competitivo (EGC), los individuos han inventado las instituciones adecuadas para restablecer, en forma aproximada, las propiedades del EGC, de modo que la comprobación empírica da el resultado observado por el positivista (p. 14).

La fortaleza del método normativo se traduce en fortaleza de la teoría neoclásica al fijarse ella misma como base para todo análisis sobre la realidad y, a la vez, que se define a ella misma como la unidad de toda teoría económica al tener la capacidad de presentar las teorías "alternativas" como casos particulares de sí misma. Se concluye que "el método normativo busca ofrecer el marco analítico y metodológico de referencia dentro del cual puede probarse, en principio, toda proposición teórica, cualquiera que sea el enfoque escogido" (Benetti, 1997, p. 15).
Ahora, debemos comprender que a partir de este método normativo los economistas pretenden interferir y transformar la realidad a través del favorecimiento del mercado y del mecanismo de precios, a pesar de que, en su fundamento, la teoría no es capaz de demostrar la eficiencia de este como mecanismo de ajuste hacia el equilibrio.

Además, es importante recalcar, como ya lo hemos señalado, que estamos hablando de una teoría abstracta cuya pretensión no es dar cuenta de la realidad. Se sigue que, teniendo en cuenta que se trata de una mala abstracción, parece ser incorrecto pretender una convergencia de la realidad económica (que está mal por no ser como el modelo), hacia un escenario que, además de irreal, no garantiza la armonía social ni la coordinación buscada por los teóricos, es decir, no resuelve el problema de la coherencia de la sociedad de mercado (Benetti, 1997).

Por esta razón, argumentan Benetti (1997), Cartelier (2018) y Cataño (2004), es necesaria una nueva teoría del valor alternativa que se fundamente en el dinero como la esencia misma de las relaciones económicas, a la vez que logre responder apropiadamente la pregunta de Smith por la coordinación de los individuos descentralizados en una sociedad mercantil.

Desde otra perspectiva, Michel Aglietta y André Orléan plantean su completa insatisfacción con esta visión del valor y de la moneda, dado que la teoría neoclásica rechaza la existencia de cualquier lazo social como parte de la explicación del problema de coordinación. Esta es explicada mediante el intercambio de bienes por parte de agentes que se encuentran aislados en sus preferencias y que no precisan, para coordinarse, otra cosa diferente a los precios de mercado; una visión atomista e individualista. La coordinación se ve limitada al ámbito mercantil y, en definitiva, no logra ser explicada por medio de dicho proceso de intercambio, sino que requiere de un mecanismo de coordinación centralizado, lo contrario a lo que buscaba describir. 
En este sentido, plantean los autores de la teoría de la regulación francesa -Orléan y Aglietta- que la moneda no puede ser simplemente un instrumento para facilitar el intercambio, no puede ser simplemente un bien que vamos a establecer convencionalmente como equivalente general para facilitar los intercambios ex-post o después de que ya las tasas de intercambio estén determinadas previamente. Básicamente, porque la moneda en su naturaleza de liquidez es esencialmente diferente de cualquier otro bien.

Lo que está detrás de la moneda es, pues, la sociedad mercantil misma previa a las relaciones individualizadas de producción e intercambio; es una forma social del valor, sin la cual no es posible coordinar decisiones mercantiles descentralizadas. La moneda es, si se quiere, el lenguaje previo que posibilita el funcionamiento del intercambio mercantil (Orléan, 2013).

Es específicamente en atención a este punto la génesis social de la moneda, que Aglietta y Orléan desarrollan su teoría de la moneda. Su propuesta consiste en entender de qué proceso social surge la moneda como expresión compartida de aquello que es lo deseado por todos en una comunidad mercantil, es decir, como expresión de la riqueza. En consecuencia, el valor económico no se explica al interior de la esfera económica exclusivamente, sino que se inserta dentro de un fenómeno mucho más global y totalizante, del conglomerado social como un todo:

nuestra concepción tiene como signo distintivo buscar captar la realidad de la moneda, no en la clásica enumeración de sus funciones, como se hace tradicionalmente, sino en su capacidad para lograr el asentimiento generalizado del grupo social en cuanto expresión adecuada del valor, del cual se desprenden sus funciones. El énfasis que se pone en esa capacidad de la moneda para lograr la unanimidad y, al hacerlo, construir la comunidad mercantil generándole su unidad y su identidad que se expresa con fuerza en fórmulas como la moneda - expresión de la sociedad como totalidad-(Orléan, 2013).

La moneda no es un elemento accesorio en la búsqueda de una explicación de la economía de mercado capitalista en la que vivimos, sino que, por el contrario, es el elemento fundante que permite explicar la coordinación descentralizada de individuos anónimos en un sistema global de mercado.

Se precisa, entonces, una teoría general que dé cuenta del vínculo entre valor-monedagénesis de la moneda-soberanía para entender 1) la génesis de la moneda y 2) cómo ella funda y actualiza la posibilidad y vigencia continuada del intercambio mercantil. Esa apuesta devendrá, pues, de la conclusión de que la moneda debe ser aceptada por un conglomerado de sujetos mercantiles y que en la aceptación de tal colectivo hay una disposición social y no psicológica (individual).

El precio es una referencia que refleja la opinión de la colectividad. Nos encontramos con una estructura especulativa, donde hay un juego de espejos en el cual cada competidor trata de leer la mente del otro. De esta manera, hay ciertos resultados empíricos que dejan ver cómo una buena proporción de los agentes convergen (Orléan, 2012). La autoreferenciación tiene la propiedad de dar una única opinión, así las demás sean muy dispersas. Se busca descubrir una emergencia, una opinión.

Para Orléan, el camino hacia la comprensión de las valoraciones y, así mismo -intuimos-, la comprensión de la coordinación de la sociedad mercantil implica una transformación radical de la ciencia económica como la conocemos hoy hacia una propuesta teórica del valor crítica y renovada. Es, entonces, necesario incorporar el análisis de las creencias que son producto de la experiencia dentro de la comprensión de la realidad económica.

Por su parte, Bruno Theret (2013), a partir de una perspectiva comparativa e histórica, pregunta por la naturaleza del dinero desde un escenario privilegiado por él, la crisis, con 
el ánimo de develar las estructuras sociales, el problema de coordinación. Así, frente a la pregunta ¿qué es el dinero? el autor responde: "la moneda es un hecho social total que garantiza la reproducción de la sociedad" (Orléan, 2013). Este hecho social total, la moneda, está soportado en el trípode deuda de vida, soberanía (legitimidad del poder político) y confianza (metódica, jerárquica y ética [Theret, 2013]). A partir de este lugar, se desprenderá la idea de Theret de que la moneda es más deuda que intercambio. Y así, la sociedad es un tejido de deudas y obligaciones que permite la reproducción social.

De lo anterior se desprende aún la pregunta por el origen del dinero. Pregunta que Theret (2015) sustenta a través de la idea de deuda de vida; concepto del cual se desprenden dos elementos: el capital de vida que permite la circulación de las deudas y las cuentas por pagar, y las relaciones sociales - somos el producto de la sociedad-de las relaciones sociales.

la deuda de vida es esencialmente una creencia, una representación, la moneda es en definitiva una expresión de la confianza o "fe social" -en términos de Marcel Mauss y François Simiand-en su capacidad para pagar y honrar las deudas por parte de una sociedad (Theret, 2015).

De esta manera queda sustentada la centralidad de la reproducción social y, en consecuencia, la vulnerabilidad de la sociedad, pues está amenazada en su existencia y su cohesión y la reproducción depende de ese conjunto de relaciones vinculadas a la deuda (Theret, 2013). De esta manera, y recogiéndose en la tradición de la sociología, la moneda es un hecho social total (Mauss, 2009), pues en su materialidad refleja elementos que son simbólicos, económicos y políticos, y es por ello que a través del análisis de la moneda es posible develar el carácter mismo de la sociedad en su conjunto y no a través únicamente de sus individuos.

Por su parte, para Cartelier (2018), en orden de avanzar hacia una teoría alternativa

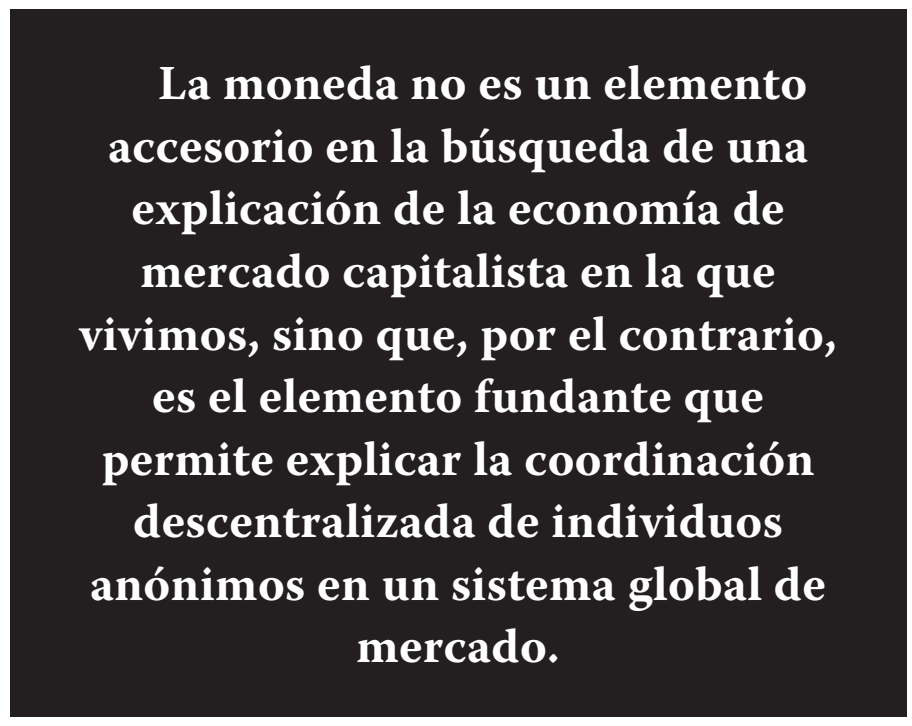

al equilibrio general, la ciencia económica debe definir sus límites y su alcance. Para esto, se propone que las relaciones económicas sean abordadas, en definitiva, como relaciones monetarias. De esta forma se reduce lo económico a lo monetario y se ofrece, para el teórico, un nuevo posicionamiento del dinero como el elemento fundamental de toda teoría y realidad económica.

Cartelier rechaza la teoría del valor de los clásicos y de la escuela neoclásica, partiendo de un análisis monetario (Schumpeter, 1954) en vez de uno real. Para él, el análisis real del dinero usa un velo que esconde la verdadera naturaleza de las relaciones económicas. Por el contrario, en el análisis monetario, el dinero es la sustancia de todas las relaciones económicas. Así mismo, critica las teorías del valor arriba mencionadas dejando en evidencia su incapacidad para pensar el dinero, recurriendo entonces al proceso de "introducir el dinero en la teoría del valor", lo que, según el autor, conduce al fracaso por cuenta de las dificultades propias del tipo de análisis adoptado. Así, el dinero es el punto de partida del análisis económico para Cartelier, es una institución dada, y a partir de entender su funcionamiento se pretende entender una economía de mercado en general y una capitalista en particular. 
Para Cartelier (2018) el dinero es el lenguaje de la economía, es el conjunto de reglas que definen la comunicación entre agentes a través de un sistema de cuentas donde se representa la actividad mercantil y, por extensión, las transferencias monetarias. Con esto Cartelier hace referencia a la existencia de un sistema de pagos como reemplazo al mundo de mercancías del modelo Arrow-Debreu, permitiéndole a la ciencia económica analizar situaciones diferentes al equilibrio.

Hemos visto, entonces, que hay una teoría económica dominante a través de la cual se nos presenta un mundo sujeto a leyes económicas frente a las cuales no hay alternativa. Que, sin embargo, la teoría neoclásica que sustenta dicha narrativa es incapaz de explicar la coordinación de acciones en una sociedad mercantil descentralizada como aquella en la que vivimos, y es incapaz, precisamente, debido a la teoría del valor que usa para explicar el funcionamiento de una economía de mercado descentralizada y que termina por acudir a mecanismos de coordinación centralizados. Se hace necesaria una teoría del valor alternativa que permita, partiendo de un análisis monetario, dar cuenta de los mecanismos que posibilitan la coordinación de agentes

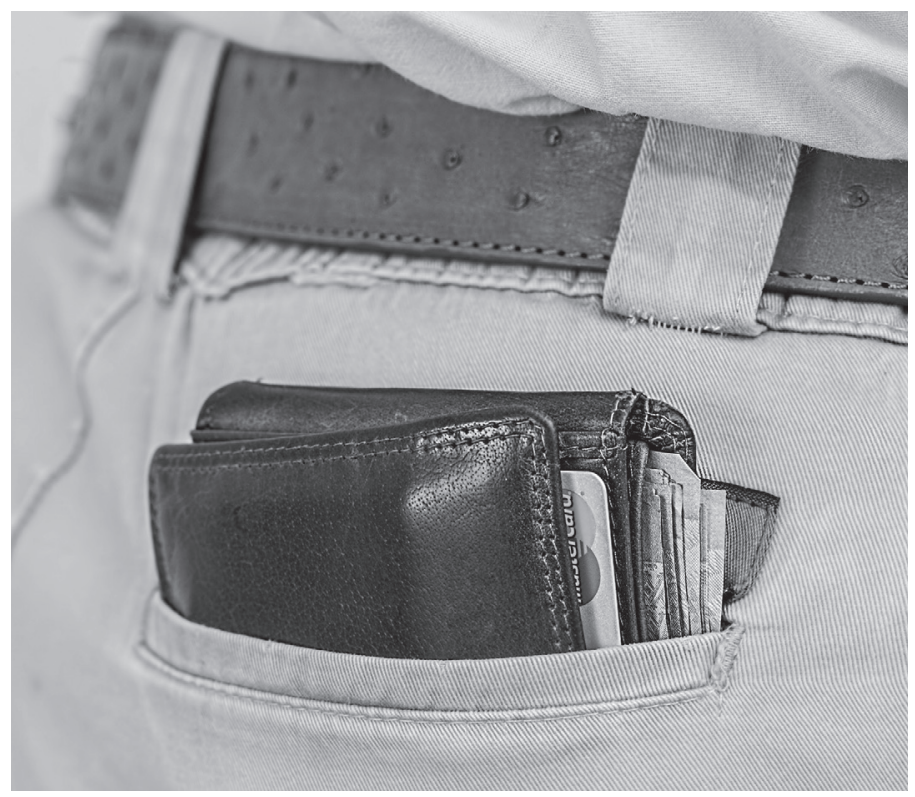

descentralizados. Por último, presentamos dos posturas frente a las teorías del valor alternativas que nos dan la posibilidad de responder a la cuestión de la coordinación en una sociedad capitalista.

Presentamos solo dos corrientes alternas frente a un mundo cada vez más amplio de posibilidades teóricas. Lo que sí queda de manifiesto es la afirmación categórica de que la narrativa fatalista es inadmisible. Sin embargo, no queda claro cuál sería el panorama si se usara una teoría del valor alternativa que sí permitiera dar cuenta de los fenómenos sobre los que versa.

Guevara (2019) asume este debate y discute la relevancia de la adopción de una u otra teoría del valor en la construcción de realidad que de ella emana. Debemos considerar entonces, por una parte, que la adopción de la teoría del valor neoclásica ha sido una elección de entre muchas otras alternativas que, como hemos visto, están mejor capacitadas para explicar la sociedad capitalista. Así mismo, debemos preguntarnos qué consecuencias ha tenido la adopción de esta teoría sobre la transformación de la realidad, en particular sobre la formulación de política pública y sus consecuencias directas sobre la vida. Por último, esta indagación nos lleva preguntar por el abanico de mundos posibles que se abre si se elige alguna heterodoxia.

Esta indagación no puede, por supuesto, dejar de lado el problema del uso funcional de la teoría elegida como sustento del discurso hegemónico para reproducir las condiciones que posibilitan su existencia. Retornando a Bourdieu (2000), las propuestas alternativas aquí reseñadas, así como las múltiples alternativas que se dejan por fuera, hacen parte de ese esfuerzo colectivo por escapar al fatalismo económico y que requieren ser integradas a procesos políticos de comprensión de los fenómenos sociales relevantes que se pretenden cambiar, como la desigualdad y las crisis, y de sus consecuencias, entendiéndolas ahora como susceptibles de ser transformadas. 


\section{Referencias}

Arrow, K. (1963). Uncertainty and the welfare economics of medical care. Chicago: Essays in the Theory of Risk-Bearing.

Benetti, C. (1997). El método normativo de la teoría económica positiva. Cuadernos de Economía. No. 26, p. 7-19.

Bourdieu, P. (2000). Una utopía razonada: contra el fatalismo económico. New Left Review en castellano, Edición (0), págs. 156-162

Cartelier, J. (2018). Money, markets and capital: the case for a monetary analysis. Abingdon: Routledge.

Cataño, J. (2004). La teoría neoclásica del equilibrio general. Apuntes críticos. Cuadernos de Economía, No. 40, p. 175-204.

Debreu, G. (1959). Theory of value. An axiomatic analysis of economic equilibrium. New Haven: Yale University Press.

De Vroey, M. (1984). La procédure de socialisation et le statut des échangistes dans trois représentations théoriques $d u$ marché. Oeconomia. No. 2.

Guevara, C. A. (2019). La moneda posible. Interrogantes epistemológicos y consecuencias de política económica de la concepción de la moneda. Tesis de investigación presentada como requisito parcial para optar al título de Doctor en Ciencias Económicas. Universidad Nacional de Colombia. Facultad de Ciencias Económicas.

Ingrao, B e Israel, G. (1990). Invisible hand. Economic equilibrium in the history of science. Cambridge: MIT Press.

Lozano, F., \& Moreno J. (2018). ¿Se comparte la misma idea al utilizar el término neoclasicismo? Cuadernos de Economía, 37(73), 25-44. doi: https://doi.org/10.15446/cuad. econ.v37n73.55414.
Mauss, M. (2009). Ensayo sobre el don. Forma y función del intercambio en las sociedades arcaicas.

Orléan, A. (2012). Knowledge in Finance: Objective value versus convention. En Richard Arena \& Agnès Festré \& Nathalie Lazaric (ed.), Handbook of Knowledge and Economics, chapter 14, Edward Elgar Publishing.

Orléan, A., \& DeBevoise, M. (2014). The Empire of Value: A New Foundation for Economics. Cambridge, Massachusetts; London, England: The MIT Press. doi:10.2307/j. ctt9qf7bb

Rebeyrol, A. (1999). La pensée économique de Walras. París: Dunod.

Schumpeter, J. A. (1954). History of economic analysis. Psychology Press.

Screpanti, E y Zamagni, S. (1995). An outline of the history of economic thought. Oxford.

Smith, A. (1958). Investigación sobre la naturaleza y causas de la riqueza de las naciones. México: Fondo de Cultura Económica.

Théret, B. (2013). La Moneda develada por sus crisis. Bogotá: Universidad Nacional de Colombia.

Théret, B. (2015). El trípode de la moneda: deuda, soberania y confianza. El laberinto de la moneda y las finanzas. La vida social de la economía (pp. 67-84) Buenos Aires: Instituto de Altos Estudios Sociales (IDAES), Universidad Nacional de San Martín.

Tobin, J. (1992). The invisible hand in modern macroeconomics. En M. Fry (editor), Adam Smith's Legacy: his place in the development of modern economics. Londres: Routledge. 